

\title{
Editorial
}

\section{The challenges of paramedic education in the new millennium: Chasing the evolution of paramedic practice}

\author{
Gavin Smith BParaStudies, GradDipEmergHIth, MEH, PhD is Associate Professor and Associate Editor ${ }^{1,2}$
}

\author{
Affiliations: \\ ${ }^{1}$ Victoria University Centre for Chronic Disease Prevention and Management, College of Health and Biomedicine \\ [Paramedicine], Melbourne, Victoria \\ ${ }^{2}$ Australasian Journal of Paramedicine
}

Across university campuses nationally, the final semester of the final year of study for hundreds of aspiring paramedics is coming to a close. With lectures completed and exams just around the corner, students are already engaged in jumping through the many hoops required to ensure their best possible chance of selection as a graduate paramedic with any one of Australia's ambulance services, and indeed international employers such as London Ambulance Service.

Some two decades ago, the education of paramedics moved from a vocational model to a higher education model, with aspirations of improvement in learning and efficiency across the sector. The practice of paramedicine has advanced rapidly (and in complexity) since that time, but questions remain as to whether paramedic education has kept pace with this evolution.

Where once paramedic education was focussed on training in essential skills coupled with a basic knowledge of pathophysiology, and subsequent application to a prescriptive set of clinical practice guidelines or protocols, the level of sophistication and expectation has grown exponentially by stakeholders (governments, the health sector and the community). Where once students practised the art of tying knots and forming slings from triangular bandages, they now learn the intricacies of pre-hospital thrombolysis and 12-lead electrocardiogram interpretation. This is a significant movement from that of training to that of education, and this paradigm shift must be accompanied by specific strategies to enable both learning and understanding $(1,2)$. These expectations also create inherent challenges for education providers, balancing the importance of incorporating new knowledge and skills into their curricula while ensuring students are capable of absorbing and understanding each aspect of learning within the same timeframe afforded them two decades ago. Thus education providers have moved beyond delivering content through traditional means (face-to-face lectures), instead utilising technologies and delivery methods designed to provide access to information, and assessment of same, outside of the traditional paradigm $(3,4)$. This has resulted in students having unprecedented access to online resources, simulation facilities and interactive computer programs that may be accessed at convenient times outside of traditional hours of learning. Although studies have identified specific benefit in simulation learning and other types of alternate learning, significant and necessary paramedic-specific research is yet to be conducted (5).

As national registration for paramedics draws closer, discussion continues about defining the new profession and the constitution of its membership. Education forms a key component of this discussion, with not only the qualification (undergraduate degree) but also its content scrutinised for industry relevance and the ability to produce 'work-ready' paramedic graduates $(6,7)$. Much has already been said about the competitive nature of clinical placement as a source of learning, and universities continue to work to identify novel means of providing students with essential clinical practicum experiences such as through high fidelity simulation and international study tour clinical placement opportunities (5). The measure of effectiveness of these learning tools is yet to be clearly defined through research, although efforts are being made in this area. 
It is possible that as the profession redefines itself, it may be necessary to look at other health professions, such as medicine, in order to identify how best to compress an ever-changing body of knowledge into a 3-year teaching program. This in itself will require significant cooperation between service providers and universities to identify expectations and deliverables regarding graduate capabilities and specific knowledge of local service provider clinical practice guidelines content. In particular, skills maintenance will be a feature and challenge in providing future 'work-ready' graduates, with research already identifying learning decay as an issue between graduation and subsequent employment (8).

This year, the annual Paramedics Australasia International Conference will be held in Melbourne in November, and will showcase the research capacity of paramedics from Australia and New Zealand, providing an annual insight into the continuing evolution of the paramedicine field. This important conference will provide an opportunity for paramedics and students to explore and understand the factors that are influencing their role in the provision of emergency health care.

The Australasian Journal of Paramedicine will continue to support and promote the knowledge and research shaping paramedic practice in order to ensure that paramedics in our region have access to the latest in developments affecting their practice. In this issue, as in a number of recent issues, we will continue to explore the questions that universities are asking of themselves and their students through the eyes of graduate and undergraduate research papers.

Gavin Smith

Associate Editor, Australasian Journal of Paramedicine

\section{References}

1. Morton J, Kloepping K, Buick JE, Todd J, Batt A. The evolution of the paramedic. Canadian Paramedicine 2015;38:22-5.

2. O'Brien K, Moore A, Dawson D, Hartley P. An Australian story: paramedic education and practice in transition. Australasian Journal of Paramedicine 2014;11:1-13.

3. Williams B, Brown T, Archer F. Can DVD simulations provide an effective alternative for paramedic clinical placement education? Emerg Med J 2008;26:377-81.

4. Hall RE, Plant JR, Bands CJ, Wall AR, Kang J, Hall C. Human patient simulation is effective for teaching paramedic students endotracheal intubation. Acad Emerg Med 2008;12:850-5.

5. Smith G. Internationalising paramedic education through clinical placement experiences. Australasian Journal of Paramedicine 2015;12:1-3.

6. McCall L, Wray N, Lord B. Factors affecting the education of pre-employment paramedic students during the clinical practicum. ibid. 2009;7:1-9.

7. Edwards D. Paramedic preceptor: work readiness in graduate paramedics. Clin Teach 2011;8:79-82.

8. Eustacia S, Schmidt TA, Mann NC, Zechnich AD. A randomized controlled trial to assess decay in acquired knowledge among paramedics completing a pediatric resuscitation course. Acad Emerg Med 2000;7:779-86. 